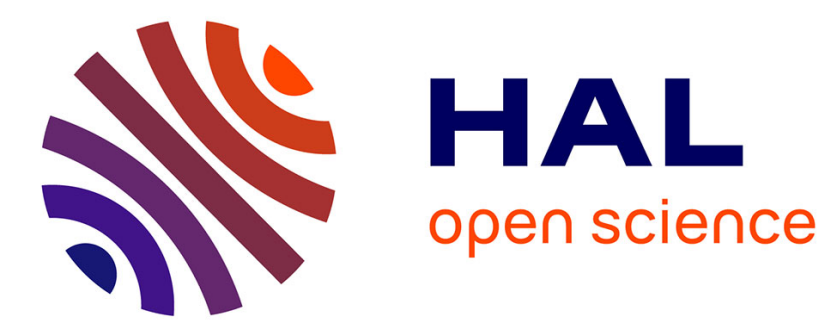

\title{
Inter-lamellar shear resistance confers compressive stiffness in the intervertebral disc: An image-based modelling study on the bovine caudal disc
}

Clayton Adam, Philippe Rouch, Wafa Skalli

\section{- To cite this version:}

Clayton Adam, Philippe Rouch, Wafa Skalli. Inter-lamellar shear resistance confers compressive stiffness in the intervertebral disc: An image-based modelling study on the bovine caudal disc. Journal of Biomechanics, 2016, 48 (16), pp.4303-8. 10.1016/j.jbiomech.2015.10.041 . hal-02454677

\author{
HAL Id: hal-02454677 \\ https://hal.science/hal-02454677
}

Submitted on 7 Feb 2020

HAL is a multi-disciplinary open access archive for the deposit and dissemination of scientific research documents, whether they are published or not. The documents may come from teaching and research institutions in France or abroad, or from public or private research centers.
L'archive ouverte pluridisciplinaire HAL, est destinée au dépôt et à la diffusion de documents scientifiques de niveau recherche, publiés ou non, émanant des établissements d'enseignement et de recherche français ou étrangers, des laboratoires publics ou privés. 


\title{
Inter-lamellar shear resistance confers compressive stiffness in the intervertebral disc: An image-based modelling study on the bovine caudal disc
}

\author{
Clayton Adam ${ }^{\mathrm{a}, \mathrm{b}, *}$, Philippe Rouch ${ }^{\mathrm{a}}$, Wafa Skalli ${ }^{\mathrm{a}}$ \\ a Institut de Biomécanique Humaine Georges Charpak, Arts et Métiers ParisTech (ENSAM), Paris, France \\ ${ }^{\mathrm{b}}$ School of Chemistry, Physics and Mechanical Engineering, Queensland University of Technology, Brisbane, Australia
}

Keywords:

Intervertebral disc

Compressive stiffness

Interlamellar interface

Shear resistance

Finite element

\begin{abstract}
A B S T R A C T
The intervertebral disc withstands large compressive loads (up to nine times bodyweight in humans) while providing flexibility to the spinal column. At a microstructural level, the outer sheath of the disc (the annulus fibrosus) comprises 12-20 annular layers of alternately crisscrossed collagen fibres embedded in a soft ground matrix. The centre of the disc (the nucleus pulposus) consists of a hydrated gel rich in proteoglycans. The disc is the largest avascular structure in the body and is of much interest biomechanically due to the high societal burden of disc degeneration and back pain. Although the disc has been well characterized at the whole joint scale, it is not clear how the disc tissue microstructure confers its overall mechanical properties. In particular, there have been conflicting reports regarding the level of attachment between adjacent lamellae in the annulus, and the importance of these interfaces to the overall integrity of the disc is unknown. We used a polarized light micrograph of the bovine tail disc in transverse cross-section to develop an image-based finite element model incorporating sliding and separation between layers of the annulus, and subjected the model to axial compressive loading. Validation experiments were also performed on four bovine caudal discs. Interlamellar shear resistance had a strong effect on disc compressive stiffness, with a $40 \%$ drop in stiffness when the interface shear resistance was changed from fully bonded to freely sliding. By contrast, interlamellar cohesion had no appreciable effect on overall disc mechanics. We conclude that shear resistance between lamellae confers disc mechanical resistance to compression, and degradation of the interlamellar interface structure may be a precursor to macroscopic disc degeneration.
\end{abstract}

\section{Introduction}

The intervertebral disc (IVD) is a unique soft tissue structure which provides support and flexibility in the axial skeleton of vertebrates. Due to the economic and social burden of disc pathologies (Hoy et al., 2014), how microstructural disease and degeneration processes in disc tissue affect the overall mechanical response of the disc is of great importance (Urban and Roberts, 2003). Previous microstructural investigations of the mammalian intervertebral disc have identified two distinct regions, the inner gel-like nucleus pulposus (NP) which is rich in proteoglycans, and the tough, fibrous, annulus fibrosus (AF) which comprises a series of concentric annular layers (lamellae), containing alternately angled collagen fibres embedded within a soft ground matrix.
The annulus confines the proteoglycan-rich nucleus gel, allowing generation of high hydrostatic pressures in response to in vivo compressive loads (Nachemson, 1960). From a structure-function perspective, the alternately aligned layers of collagen fibres in adjacent lamellae serve to resist both internal NP pressures during joint loading and torsion through fibre tension. However, what is not currently clear is the role of the interface between adjacent lamellae, and the extent to which interface mechanics governs overall disc behaviour. The importance of interlamellar mechanics is further highlighted by the findings of Marchand and Ahmed (1990) in which the authors reported a high proportion (40-50\%) of circumferentially discontinuous lamellae in human lumbar IVDs. A substantial proportion of the lamellae is therefore not rings, but circumferentially incomplete curved sheets, which raises the question as to how the interface allows shear stress transfer between discontinuous lamellae.

Despite several careful investigations of the microanatomy of the interlamellar interface, existing knowledge on interlamellar mechanics remains sparse and somewhat contradictory. Lamellar 
layers have been readily separated in several dissection studies (Marchand and Ahmed, 1990; Holzapfel et al., 2005), and Bruehlmann et al. (2004) show evidence of inter-lamellar sliding ('discrete deflection') in the inner $\mathrm{AF}$ of the bovine disc under $8^{\circ}$ flexion, both of which suggest that interlamellar interfaces are mobile. On the other hand, the presence and extent of the interlamellar fibre network (Yu et al., 2005, 2007; Pezowicz et al., 2006; Schollum et al., 2009) suggests a structural role in limiting slip between lamellae, and Smith et al. (2008) demonstrated that the inter-lamellar elastin network of human lumbar discs confers substantial tensile stiffness of the interface. Two quantitative studies on the shear resistance of the interlamellar interface are those of Michalek et al. (2009) who reported that sliding did not occur between AF layers in bovine discs, and Gregory et al. (2011), who found substantial interlamellar load carrying ability in porcine cervical discs. We note also the T-peel tests of Gregory et al. (2012) who reported $\sim 30 \%$ higher peel strength in the superficial annulus of human lumbar discs than in the inner annulus.

The aim of this study was to investigate the extent to which interlamellar interface mechanics governs overall disc resistance to compression, the fundamental loading state to which the disc is subjected in vivo. This aim was pursued through the development of an image-based micro-scale FE model of the disc incorporating both interlamellar sliding and separation.

\section{Methods}

The FE model developed in this study was based upon the bovine tail disc. Bovine tails provide a consistent and readily available source of tissue with AF collagen content comparable to human discs (Showalter et al., 2012), and there is a growing body of basic science studies on the microarchitecture and mechanics of the bovine tail disc (Bruehlmann et al., 2004; Pezowicz et al., 2006; Yu et al., 2007; Michalek et al., 2009).

A single motion segment (second intervertebral joint) of an adult bovine tail was freshly obtained from a local butcher and frozen at $-20^{\circ} \mathrm{C}$ prior to use. Lateral and frontal radiographs were taken for subsequent endplate curvature and disc height measurement. The excised motion segment was fixed, dehydrated and embedded en bloc in Spurr resin (Electron Microscopy Sciences, Hatfield, PA, USA). A thick $(\sim 300 \mu \mathrm{m})$ mid-height transverse section was prepared by cutting and milling the embedded disc, before mounting to a large format glass microscope slide. By using a thick section, the need for staining to enhance collagen birefringence in polarized light could be avoided. This was desirable in order to avoid potential artefacts due to uneven staining (Kiraly et al., 1997).

The transverse disc section was imaged using a custom polarized light imaging system with white LED (6500 K colour temperature, Lightune Inc, France) illumination, and crossed circular polarizer and analyzer (Edmund Optics Ltd., Europe) positioned beneath and above the specimen. A custom mounted tube-scope was equipped with a $4 \times$ finite conjugate plan objective (Edmund Optics Ltd., Europe) and a five megapixel colour CMOS microscope camera (DCM-510, ScopeTek, China). The computer controlled imaging system was used to take a regularly spaced grid of microscope images which covered the entire disc cross-section, and these were post-stitched using the algorithm of Preibisch et al. (2009) provided as part of the Fiji distribution of the ImageJ software (NIH, USA).

Disc microstructure (lamellar count, thickness and continuity) in the stitched transverse plane image was measured as follows. Firstly, the origin of radial coordinates was defined by a vector oriented from the centroid of the disc cross-section toward the ventral edge of the disc. Angles were defined relative to this axis, so that the left and right lateral directions were at $+90^{\circ}$ to the ventral axis respectively, and the dorsal (posterior) direction was at $180^{\circ}$. Using this polar coordinate system, lamellar number and thickness were counted and measured along axes from the disc centroid at angles of $0^{\circ}$ (ventral), $\pm 90^{\circ}$ (left and right lateral) and $180^{\circ}$ (dorsal). Lamellar layers were counted from the outer to the inner annulus (Fig. 1). In the transverse cross-section, a lamellar origin or termination was defined as a clearly visible ' $\mathrm{Y}$ ' junction (Fig. 2). Lamellar discontinuities were manually marked by measuring the $x, y$ coordinates of the tip of each discontinuity using the Image point measurement tool. All measurements were performed by a single observer.

The measurements described above were used to generate an image-based FE model to study the effects of inter-lamellar interface mechanics and lamellar discontinuity on disc compression resistance. Firstly, the external AF diameter from the transverse section was taken as the outer diameter of the model. Next, the mean thicknesses of the outer, middle and inner third of the lamellar layers in the embedded disc cross-section at mid-height were used to define the corresponding

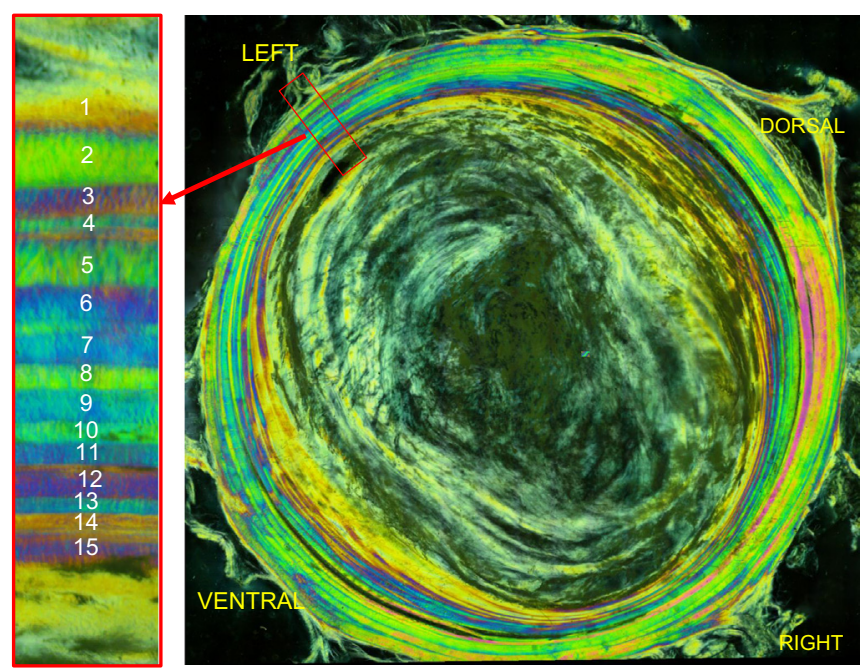

Fig. 1. Stitched polarized light image of entire transverse cross-section of the bovine intervertebral disc used for FE model generation. The inset at left shows an example of lamellar counting from outer to inner edges of the annulus fibrosus.

lamellar thicknesses in the FE model. Thirdly, the endplate curvatures and disc height measured from lateral and frontal radiographs of the motion segment taken prior to embedding and sectioning were used to define the endplate curvature and initial disc height of the FE model. A 3D FE model of $1 / 8$ of the disc was then generated (one quarter of the transverse plane cross-section assuming mid-sagittal and mid-coronal plane symmetry based on the high circularity of the bovine tail disc in this plane, and assumed superior-inferior symmetry about the midtransverse plane of the disc).

Each lamella was meshed individually, firstly with 3D continuum solid elements to represent the ground matrix, and secondly with an embedded layer of ABAQUS 'rebar' elements to represent the collagen fibre bundles within each layer. The collagen fibre alignments in successive lamellar layers were alternated between $\pm 30^{\circ}$ to the transverse plane (Marchand and Ahmed, 1990). The thickness and spacing of collagen fibre bundles within each lamellae were also taken from Marchand and Ahmed (1990), with a cosine correction to account for the orientation of the sectioning plane relative to the fibre orientation (fibre bundle crosssectional area $3.212 \times 10^{-2} \mathrm{~mm}^{2}$, spacing $0.23 \mathrm{~mm}$ ). The NP was meshed using 3D solid elements. Material properties for the collagen fibres and AF ground substance were based on prior literature, and the Mooney-Rivlin constants for the NP were prescribed to be an order of magnitude less stiff than the AF ground substance while maintaining near-incompressible material behaviour $(D=0.3$ corresponding to $\mu_{\text {eff }}=0.487$ ) (Table 1 ).

In order to investigate the effect of inter-lamellar cohesion and lamellar discontinuity, separate contact interfaces were defined between every pair of adjacent lamellae in the model. These interfaces could either be 'tied' (no relative slip and no separation), or allowed to slip/separate. Furthermore, in order to simulate the introduction of several circumferentially discontinuous lamellae in the model, the $\Delta x=0$ boundary condition at the mid-sagittal symmetry plane was released for three of the lamellae in the model (the 3rd, 8th and 13th lamellae from the outer annulus) thus allowing circumferential retraction under load. Six different sets of interlamellar interface conditions were simulated in all (Table 2).

The model was loaded in axial compression to $400 \mathrm{~N}$ using a central node which was constrained to all of the nodes on the upper surface of the mode (corresponding to the vertebral endplate). The displacement at $10 \mathrm{~N}$ of compression was used as the reference (zero) displacement for stiffness calculation. All FE modelling was performed using ABAQUS (v6.11, Dassault Systèmes, Paris, France) using a quasi-static solution procedure with large displacement solver. Mesh sensitivity analysis was performed using an intact NP model with Interface Condition 1 from Table 2 .

A small series of bovine tail disc compression experiments were then performed for comparison with the FE model predictions. Two adult bovine tails were freshly obtained from a local butcher and immediately frozen at $-20^{\circ} \mathrm{C}$. Prior to dissection, specimens were defrosted overnight. Once thawed, the upper two caudal discs from each tail were carefully dissected to expose the vertebral column, leaving intervertebral discs intact. The first, second and third caudal vertebrae were sectioned transversely at mid-height, yielding two intact motion segments per tail for subsequent biomechanical testing. The cut ends of the adjacent vertebral bodies were embedded in polymethylmethacrylate. After curing, the test specimens underwent uniaxial compression in an Instron universal testing machine (Mode 5566, Instron, UK). Each specimen was first subjected to three cycles of compressive preload from 10 to $50 \mathrm{~N}$, followed by a single cycle of compressive load to a maximum of $400 \mathrm{~N}$. The pre-cycles and the main load were all performed at a 

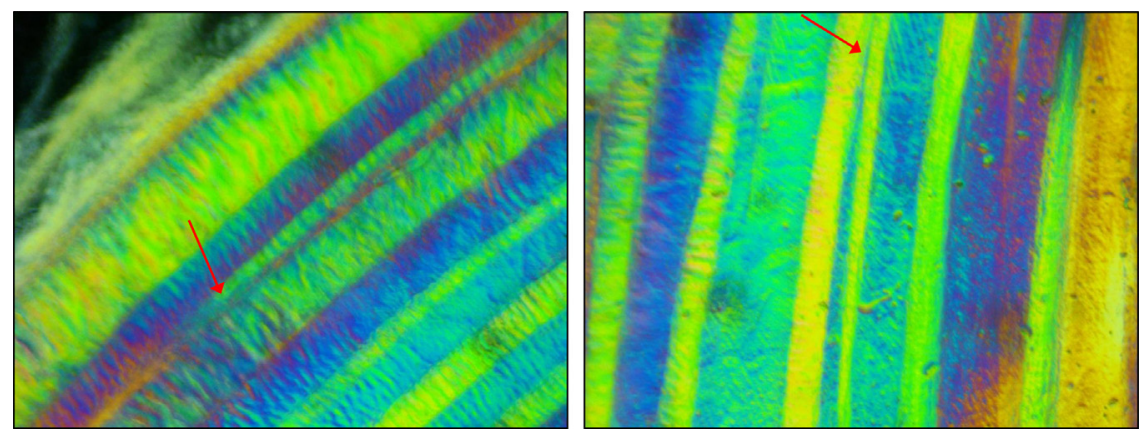

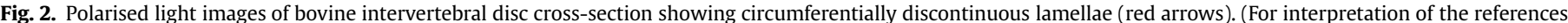
to colour in this figure legend, the reader is referred to the web version of this article.)

Table 1

Material properties and mesh details for the FE model.

\begin{tabular}{|c|c|c|c|}
\hline Component & Constitutive model & Material parameters & ABAQUS element type \\
\hline Lamellar ground substance & $\begin{array}{l}\text { Hyperelastic (Mooney-Rivlin strain energy } \\
\text { potential) }\end{array}$ & $\begin{array}{l}C_{01}=0.7, C_{10}=0.2, D=0\left(\mu_{\mathrm{eff}}=0.5\right) \text { (Natali and } \\
\text { Meroi, } 1990)\end{array}$ & $\begin{array}{l}\mathrm{C} 3 \mathrm{D} 8 \mathrm{H} \text { ( } 8 \text { node continuum hybrid } \\
\text { formulation) }\end{array}$ \\
\hline Collagen fibres & Linear elastic & $\begin{array}{l}E=500 \mathrm{MPa}, \nu=0.3 \text { (no compression) (Ueno } \\
\text { and Liu, 1987) }\end{array}$ & $\begin{array}{l}\text { SFM3D4R ( } 4 \text { node embedded rebar surface } \\
\text { elements) }\end{array}$ \\
\hline Nucleus pulposus & $\begin{array}{l}\text { Hyperelastic (Mooney-Rivlin strain energy } \\
\text { potential) }\end{array}$ & $C_{01}=0.07, C_{10}=0.02, D=0.3\left(\mu_{\mathrm{eff}}=0.487\right)$ & $\begin{array}{l}\text { C3D8H (8 node continuum hybrid } \\
\text { formulation) }\end{array}$ \\
\hline
\end{tabular}

Table 2

FE Models used to explore the effect of interlamellar interface cohesion and shear resistance on disc compression response.

\begin{tabular}{|c|c|c|c|}
\hline Interface Condition & Circumferential continuity of lamellae? & Inter-lamellar slip allowed? & Inter-lamellar separation allowed? \\
\hline 1 & All & No (tied interface) & No \\
\hline $1 b$ & (as above with $4.5 \mathrm{~mm}$ axial relief hole in $\mathrm{NP}$ ) & & \\
\hline 2 & All & Yes, Coulomb friction $(\mu=1)$ & No \\
\hline 3 & All & Yes, frictionless $(\mu=0)$ & No \\
\hline $3 b$ & (as above with $4.5 \mathrm{~mm}$ axial relief hole in $\mathrm{NP}$ ) & & \\
\hline 4 & All & Yes, frictionless $(\mu=0)$ & Yes \\
\hline 5 & $20 \%(3 / 15)$ of lamellae discontinuous & Yes, Coulomb friction $(\mu=1)$ & No \\
\hline 6 & $20 \%(3 / 15)$ of lamellae discontinuous & Yes, frictionless $(\mu=0)$ & No \\
\hline
\end{tabular}

loading rate of $100 \mathrm{~N} / \mathrm{min}$. Specimens were kept moist throughout testing, and all tests were performed at room temperature.

After the first test, each specimen was removed from the testing machine and a $4.5 \mathrm{~mm}$ drill bit was used to drill a relief hole axially through the superior adjacent vertebra and into the nucleus pulposus region of the disc. This did not achieve a full nucleotomy, but allowed nucleus material to extrude through the endplate and into the adjacent vertebra body during compression, therefore reducing the accumulation of nucleus pressure in the subsequent 'relief hole' compression test, which was performed immediately after the intact test described above. When modelling the relief hole compression test, a $4.5 \mathrm{~mm}$ diameter cylinder of NP tissue was removed from the FE model, and remaining NP material was able to bulge into this hole during compression.

Force and displacement were logged at $10 \mathrm{~Hz}$ during all testing and subsequently exported for analysis. Force vs displacement plots were generated from the main loading cycle over a range from 10 to $400 \mathrm{~N}$ (i.e. zero displacement was considered to be the value at $10 \mathrm{~N}$ for all tests).

\section{Results}

The overall dorsal-ventral width of the outer annulus fibrosus of the imaged disc used to generate the FE model was $22.81 \mathrm{~mm}$, and the lateral width was $22.96 \mathrm{~mm}$ (circularity to within $0.7 \%$ ). The transverse cross-sectional area of the disc was $411 \mathrm{~mm}^{2}$. There were 15, 11, 15 and 16 lamellae through the annulus thickness at the dorsal, ventral, left and right locations respectively, with individual and mean lamellar thicknesses for each region of the disc given in Table 3. The simplified FE model comprised 15 lamellar layers, with the thickness of each of the five layers in the outer, middle, and inner third respectively (Table 3 ). Thus the innermost (16th) lamellar layer of the actual bovine tail disc was not included in the FE model, and layers 12-15 at the ventral annulus were present in the FE model but not in the actual disc. The mean lamellar thickness for all layers at all locations was $152 \mu \mathrm{m}$. The minimum (at the centre of the nucleus) height of the modelled disc was $4.5 \mathrm{~mm}$, compared to $3.9 \pm 1.3 \mathrm{~mm}$ for the experimentally tested discs. Due to the convexity of the bovine caudal vertebral body, disc height increases to $\sim 7 \mathrm{~mm}$ at the annulus.

Only 25 discontinuities were identified in the entire disc. In order to be consistent with the proportions of discontinuous lamellae reported by Marchand and Ahmed using their peeling technique, we consider that each single discontinuity would have led to an incomplete layer being peeled. Therefore when using the same $20^{\circ}$ circumferential slices of annulus as reported by Marchand and Ahmed, 0/11 ventral lamellae were discontinuous, 1/15 dorsal, 3/15 left lateral and 4/16 right lateral, for an overall $8 / 57=14 \%$ discontinuous lamellae.

Compressive force vs displacement curves from the FE model were compared with the experimentally tested discs for two interface conditions; (i) tied, and (ii) free sliding ( $\mu=0$ ) (Fig. 3). The mean stiffness for the experimentally tested discs was $422 \pm 35 \mathrm{~N} / \mathrm{mm}$ for the intact discs, and $397 \pm 30 \mathrm{~N} / \mathrm{mm}$ after the 
Table 3

Individual lamellar count and thickness (in $\mu \mathrm{m}$ ) for the bovine tail disc used to generate the finite element model. Note that lamella 1 is the outermost layer.

\begin{tabular}{llllll}
\hline Lamella \# & Dorsal & Ventral & Left & Right & Mean \\
\hline 1 & 133 & 316 & 185 & 222 & 214 \\
2 & 340 & 260 & 106 & 113 & 205 \\
3 & 130 & 127 & 69 & 57 & 95 \\
4 & 158 & 141 & 42 & 39 & 95 \\
5 & 215 & 126 & 175 & 176 & 173 \\
Outer third & Mean 157 & & SD 83 & & \\
6 & 225 & 163 & 153 & 138 & 170 \\
7 & 242 & 147 & 164 & 53 & 152 \\
8 & 207 & 295 & 79 & 116 & 174 \\
9 & 256 & 149 & 143 & 88 & 159 \\
10 & 200 & 225 & 80 & 134 & 160 \\
Middle third & Mean 163 & & SD 65 & & \\
11 & 186 & 254 & 85 & 81 & 152 \\
12 & 179 & & 122 & 102 & 134 \\
13 & 149 & & 69 & 110 & 109 \\
14 & 225 & & 90 & 57 & 124 \\
15 & 254 & & 112 & 67 & 144 \\
Inner third & Mean 134 & & SD 66 & & \\
16 & & & & 130 & \\
\hline
\end{tabular}

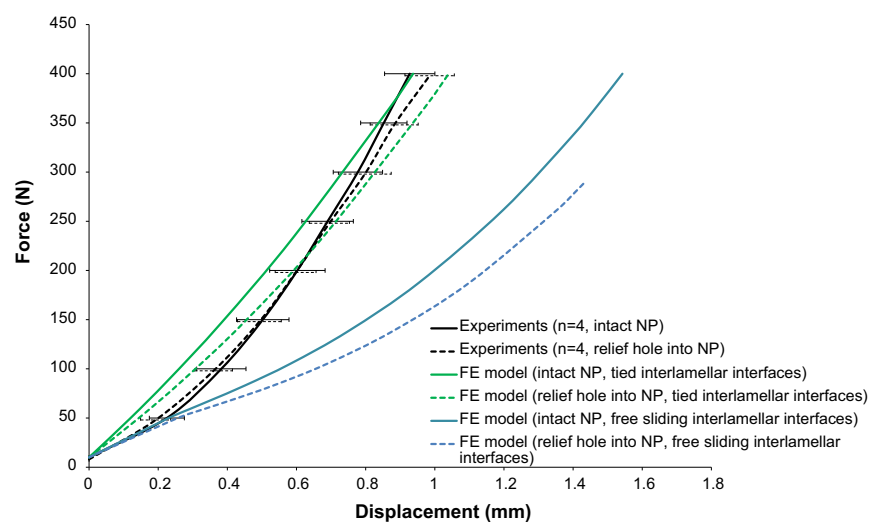

Fig. 3. Comparison of FE model and axial compression experiments for four bovine tail discs (experimental error bars \pm 1 std). Solid lines show the response of the intact disc, and dotted lines show the response of the same disc after drilling of the axial relief hole. The FE models shown are for interface conditions 1, 1b, 3 and 3b.

$4.5 \mathrm{~mm}$ relief holes. This drop in stiffness of $-5.8 \%$ was statistically significant $(P=0.042$, paired $t$-test). As is evident from Fig. 3 , the FE model with tied interlamellar interfaces fell within the range of the experimental data, with a predicted secant stiffness of $416 \mathrm{~N} / \mathrm{mm}$ for the intact model, and $375 \mathrm{~N} / \mathrm{mm}$ for the $4.5 \mathrm{~mm}$ relief hole model. The tied interface model stiffness was therefore $1.5 \%$ lower than the mean experimentally measured stiffness, and the $-9.9 \%$ change in FE model stiffness with addition of the axial relief hole was $4 \%$ greater than the experimental change in stiffness $(-5.8 \%)$.

Table 4 gives the results of the different FE model interface conditions from Table 2. The highest stiffness occurred in the model with tied interfaces. Allowing inter-lamellar slip resulted in a substantial drop in the predicted compressive stiffness in all cases, to around $340 \mathrm{~N} / \mathrm{mm}$ (20\% drop in stiffness) in the case where a friction coefficient of 1.0 was prescribed between lamellae (interface conditions 2,5 ), and to $250 \mathrm{~N} / \mathrm{mm}$ ( $40 \%$ drop in stiffness) in the cases where the sliding interfaces were frictionless (interface conditions 3, 4, 6). Deformation patterns for selected FE models are compared between the unloaded state (Fig. 4a and d) and $400 \mathrm{~N}$ compression in Fig. 4. Note the bulging of NP material into the $4.5 \mathrm{~mm}$ relief hole under compressive loading (compare Fig. $4 \mathrm{~d}$ and e), the difference in AF deformation between tied and
Table 4

Predicted compressive stiffness for varying inter-lamellar interface conditions ( $\mu$ is friction coefficient). Values are secant stiffness between 10 to $400 \mathrm{~N}$ compression. All FE models had intact NP unless otherwise stated.

\begin{tabular}{|c|c|c|}
\hline $\begin{array}{l}\text { Interlamellar interface } \\
\text { condition }\end{array}$ & $\begin{array}{l}\text { FE model axial com- } \\
\text { pression stiffness }(\mathrm{N} / \\
\mathrm{mm})\end{array}$ & $\begin{array}{l}\text { \% change in stiffness } \\
\text { relative to Interface } \\
\text { Condition } 1 \text { with intact } \\
\text { NP }\end{array}$ \\
\hline 1 (no slip, no separation) & 416 & - \\
\hline $\begin{array}{l}\text { 1b (as above with } 4.5 \mathrm{~mm} \\
\text { axial relief hole in NP }\end{array}$ & 375 & $-10 \%$ \\
\hline $2(\mu=1$, no separation $)$ & 339 & $-19 \%$ \\
\hline $3(\mu=0$, no separation $)$ & 253 & $-39 \%$ \\
\hline $\begin{array}{l}\text { 3b (as above with } 4.5 \mathrm{~mm} \\
\text { axial relief hole in NP) }\end{array}$ & 196 & $-53 \%$ \\
\hline $4(\mu=0$, separation allowed $)$ & 252 & $-40 \%$ \\
\hline $\begin{array}{l}5(\mu=1 \text {, no separation, } \\
3 \text { discontinuous lamellae) }\end{array}$ & 337 & $-19 \%$ \\
\hline $\begin{array}{l}6(\mu=0, \text { no separation, } \\
3 \text { discontinuous lamellae })\end{array}$ & 251 & $-40 \%$ \\
\hline
\end{tabular}

freely sliding interlamellar interface conditions (compare Fig. $4 \mathrm{~b}$ and $\mathrm{c}$ ), and the retraction of circumferentially discontinuous lamellae under load (Fig. 4f). The compressive stiffness predicted by the FE model was insensitive to mesh size over the range of AF element sizes $300-750 \mu \mathrm{m}$ (less than $1 \%$ change in predicted compressive stiffness across this range).

In terms of predicted disc tissue mechanics under the $400 \mathrm{~N}$ compressive load, the nucleus pulposus hydrostatic pressures predicted in the tied interface model (Table 2 condition 1 ) and the frictionless interface model (Table 2 condition 3 ) were $0.9 \mathrm{MPa}$ and 1.22 MPa respectively. The predicted maximum principal strains in the annular collagen fibres were $2.1 \%$ for the tied interface model and $5.6 \%$ for the frictionless interface model. Both of these quantities are indicative of the reduction in annular load carrying ability which occurs when interlamellar shear resistance is removed.

\section{Discussion}

This work was motivated by the desire to better understand the significance of the interlamellar interface of the IVD in terms of its role in conferring macro-scale disc compression resistance (Goel et al., 1995). To our knowledge this is the first disc FE model to incorporate discontinuous interlamellar sliding and separation, although we note that continuum constitutive models incorporating interlamellar interactions have recently been developed (Guerin and Elliott, 2007; Nerurkar et al., 2011; Labus et al., 2014). We found that disc compressive stiffness is relatively sensitive to interlamellar shear resistance, but not sensitive to interlamellar cohesion. With no other changes in geometry or material properties, allowing adjacent lamellae to slide freely across each other reduces disc stiffness by $40 \%$ compared to the case where interlamellar interfaces are bonded.

It is important to acknowledge that the actual degree of interlamellar shear resistance in the intervertebral disc remains unclear. The bovine tail disc experiments of Michalek et al. (2009) did not find any sliding between layers, yet those of Bruehlmann et al. (2004) found discrete deflection between inner AF layers. Experimental investigation of inter-lamellar shear resistance should therefore be a priority for future micro-mechanical investigations of the disc. In our study, the fact that Interface Condition 1 (tied interfaces) matched closely with experimental results might be interpreted as saying that the bovine tail disc has a high degree of inter-lamellar shear resistance. However this conclusion is only tentative, because although the geometry of the current 


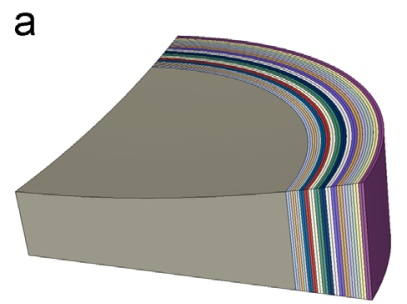

b

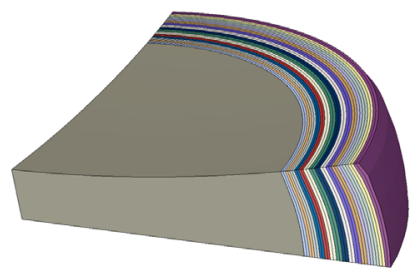

e

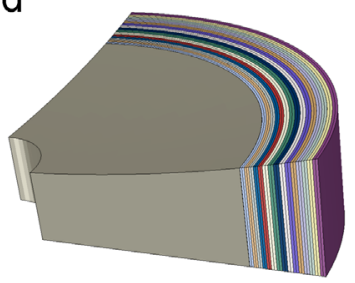

C
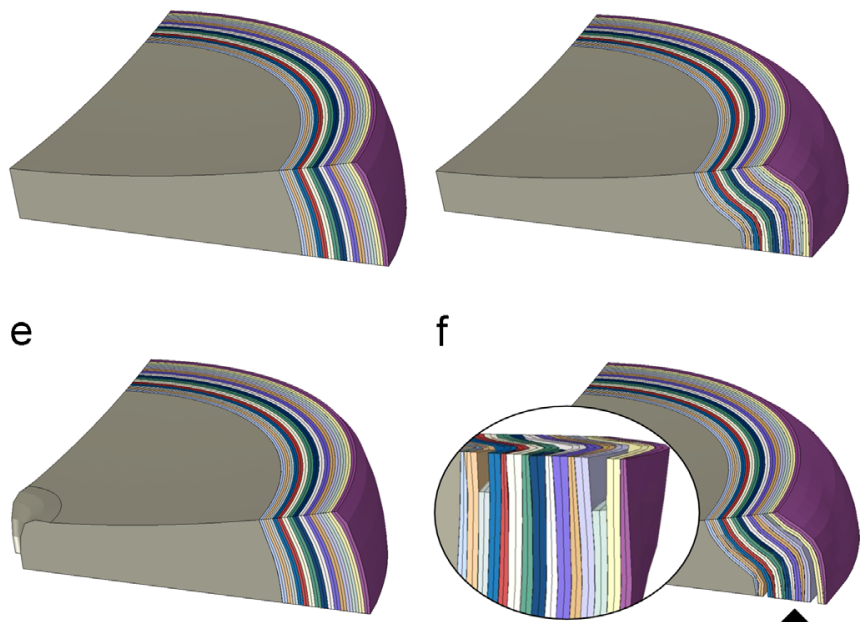

f

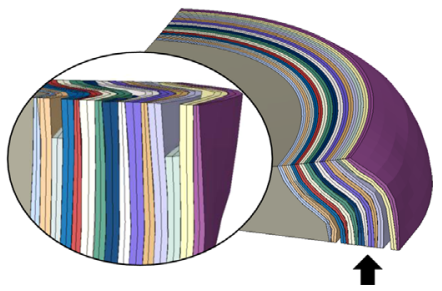

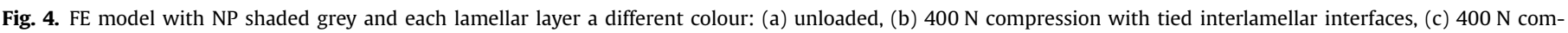

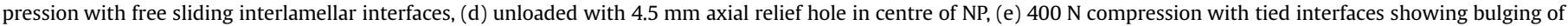

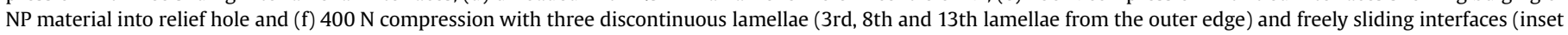
shows circumferential retraction of lamellae-viewed in the direction of the arrow).

model was based on actual bovine tail disc microstructure, the material properties and collagen fibre density/orientation were based on other literature, so were not specific to the discs tested in this study. In particular, the predicted compressive force vs displacement response is very sensitive to the hyperelastic compressibility coefficient ( $D$ in Table 1 ) of the NP elements. If there actually is a high degree of inter-lamellar bonding then incorporating inter-lamellar sliding in FE models of the disc may not be necessary, but the counterevidence of inter-lamellar sliding provided by Bruehlmann et al. (2004) cannot be discounted, nor can the possibility that breakdown of the inter-lamellar network is an important part of disc degeneration in humans. For these reasons, the standard approach of embedding alternating layers of crisscrossed annular fibre bundles into continuum FE models of the disc may be a necessary, but not sufficient step in properly representing the microstructural basis of disc mechanics.

With regard to the interface conditions used in the FE Model, we defined Coulomb friction between lamellar layers in order to simulate a finite degree of shear resistance at the interface. This approach was not intended to imply that actual friction is the primary mechanism of inter-lamellar shear resistance, but rather to provide a numerically convenient means to represent finite shear resistance which may be present at the interface. The significance of a unity friction coefficient (interface conditions 2 and 5) is that it provides an intermediate level of interlamellar shear resistance, such that shear stresses cannot exceed normal stresses across the interface. As already stated, interlamellar shear resistance has not been quantified in the disc to date, and therefore this modelling approach is intended as a starting point, with acknowledgement that other approaches are possible.

There are several limitations to this study. Firstly, since a transverse plane disc cross-section was used to generate the image-based FE model, no information on longitudinal variations in lamellar thickness, radial location or number was contained in this image. Thus each lamella is modelled as a thin hollow cylinder whose cross-section is taken from the mid-transverse plane polarized light micrograph. Disc bulge can occur subsequently under load, but the initial lamellar architecture is assumed to be vertical-sided. Having said this, the longitudinal polarized light micrograph in Fig. 5 (of another bovine tail disc) demonstrates than the vertical-sided approximation of lamellae used in this study is not unreasonable. There is some curvature of the inner lamellar layers; but the outer two thirds of layers run in nearly straight lines from endplate to endplate. Secondly, the cartilaginous

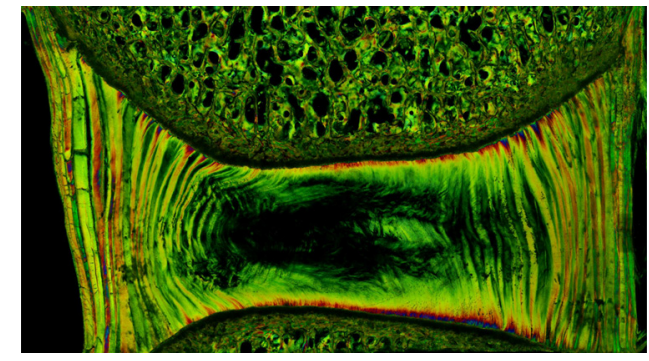

Fig. 5. Longitudinal plane polarized light micrograph showing approximately vertical orientation of lamellar walls in the unloaded bovine tail disc.

endplate in the bovine tail disc is thin $(\sim 0.2 \mathrm{~mm})$ and was not incorporated in the model, although we suggest that future iterations of the model could assess model sensitivity to the presence of a thin cartilaginous endplate. Thirdly, the actual lamellar thicknesses measured in Table 3 are averaged (both circumferentially and according to annular region) when generating the FE model, thus the influence of heterogeneities in individual lamellar thicknesses is not captured in the current model.

In addition to the geometric simplifications introduced in the FE model, it is known that alcohol dehydration during the embedding process can cause shrinkage in soft tissues. Since the degree of shrinkage was unknown, we did not attempt any geometric normalization of disc compressive stiffness, although we note that such approaches have been usefully employed for comparing different sized specimens by other authors (Beckstein et al., 2008; Showalter et al., 2012). However, we did attempt to minimize shrinkage by performing the dehydration and embedding en bloc with the disc still attached to the neighbouring vertebrae (Ferguson et al., 1999). Fig. 1 shows evidence of shrinkage-induced delamination between lamellar layers, and careful inspection of Fig. 1 shows that there are some regions of distinct colour (e.g. between lamellae 6 and 7) which could either be another thin lamella or a partial delamination between layers (where the interference colour is distinct due to the reduced collagen density in the partially delaminated layer). In the current study we have assumed these thin layers to be partial delaminations; however we acknowledge that this is a subjective decision.

With regard to the assumption of linear elastic collagen fibre constitutive behaviour in the current study, many collagenous tissues 
exhibit a crimp or waviness in their relaxed configuration and therefore offer very little resistance to tension until 3-4\% strain. However the (externally) unloaded intervertebral disc annulus is subject to prestrain due to swelling of the hydrophilic proteoglycans in the nucleus, and it stands to reason that collagen crimp in annular layers may be pre-stretched to some extent. Indeed close inspection of the magnified inset in Fig. 1 shows evidence of collagen crimp in a few lamellar layers but not in others. Therefore we suggest that the assumption of linear collagen material response can be at least partially justified on this basis.

Interestingly, introducing either inter-lamellar separation or circumferentially discontinuous lamellae into the model (interface conditions 4, 5 and 6) had little effect on predicted compressive stiffness. In the case of inter-lamellar separation, we suggest that this occurred because when the annulus is internally loaded by nucleus pressure, the inter-lamellar interfaces transmit positive pressure which keeps them in contact, even in the absence of cohesion. This raises the question as to whether inter-lamellar bonds become more important in the case of reduced nucleus pressure. Table 4 provides some support for this; when the interlamellar interface is tied, the axial relief hole only reduced disc stiffness by $10 \%$ (interface conditions 1 vs $1 \mathrm{~b}$ ), whereas when the interlamellar interfaces were freely sliding, the axial relief hole reduced predicted disc stiffness by 25\% (interface conditions 3 vs $3 b)$. So loss of nucleus pressure is relatively more important in discs with low interlamellar shear resistance. With regard to the negligible effect of introducing discontinuous lamellae into the model, this appears to be due to the principal membrane stress direction in the lamellae being axial rather than circumferential, therefore axial (endplate-to-endplate) continuity of lamellae is more important than circumferential continuity. Our method of representing circumferentially discontinuous lamellae did not attempt to reproduce the actual discontinuity locations measured, but rather took advantage of the symmetry boundary condition to allow retraction in the three lamellae released in interface conditions 5 and 6 . The decision to introduce three discontinuous lamellae into the FE model $(3 / 15=20 \%)$ was based on the finding of $14 \%$ discontinuous lamellae in the polarized light micrograph. This is less than half of the number of circumferential discontinuities (40-50\%) reported in the human IVD by Marchand and Ahmed (1990), suggesting that perhaps lamellar continuity alters with age and/or between species.

\section{Conflict of interest statement}

None of the authors have any financial and personal relationships with other people or organizations that could inappropriately influence the work described in this manuscript.

\section{Acknowledgements}

This research was supported by a Marie Curie International Incoming Fellowship within the 7th European Community Framework Programme (FP7-PEOPLE-2010-IIF 274964 DiscSim). The authors wish to acknowledge the technical assistance of Mr. Sylvain Persohn and Ms. Maria Jeyasankar.

\section{References}

Beckstein, J.C., Sen, S., Schaer, T.P., Vresilovic, E.J., Elliott, D.M., 2008, Comparison of animal discs used in disc research to human lumbar disc: axial compression mechanics and glycosaminoglycan content. Spine 33 (6), E166-E173.

Bruehlmann, S.B., Matyas, J.R., Duncan, N.A., 2004. ISSLS prize winner: collagen fibril sliding governs cell mechanics in the anulus fibrosus: an in situ confocal microscopy study of bovine discs. Spine 29 (23), 2612-2620.

Ferguson, S.J., Bryant, J.T., Ito, K., 1999. Three-dimensional computational reconstruction of mixed anatomical tissues following histological preparation. Med. Eng. Phys. 21 (2), 111-117.

Goel, V.K., Monroe, B.T., Gilbertson, L.G., Brinckmann, P., 1995. Interlaminar shear stresses and laminae separation in a disc. Finite element analysis of the L3-L4 motion segment subjected to axial compressive loads. Spine 20 (6), 689-698.

Gregory, D.E., Bae, W.C., Sah, R.L., Masuda, K., 2012. Anular delamination strength of human lumbar intervertebral disc. Eur. Spine J. 21 (9), 1716-1723.

Gregory, D.E., Veldhuis, J.H., Horst, C., Wayne Brodland, G., Callaghan, J.P., 2011 Novel lap test determines the mechanics of delamination between annular lamellae of the intervertebral disc. J. Biomech. 44 (1), 97-102.

Guerin, H.L., Elliott, D.M., 2007. Quantifying the contributions of structure to annulus fibrosus mechanical function using a nonlinear, anisotropic, hyperelastic model. J. Orthop. Res. 25 (4), 508-516.

Holzapfel, G.A., Schulze-Bauer, C.A., Feigl, G., Regitnig, P., 2005. Single lamellar mechanics of the human lumbar anulus fibrosus. Biomech. Model Mechanobiol. 3 (3), 125-140.

Hoy, D., March, L., Brooks, P., Blyth, F., Woolf, A., Bain, C., Williams, G., Smith, E., Vos T., Barendregt, J., Murray, C., Burstein, R., Buchbinder, R., 2014. The global burden of low back pain: estimates from the Global Burden of Disease 2010 study. Ann. Rheum. Dis. 73 (6), 968-974.

Kiraly, K., Hyttinen, M.M., Lapvetelainen, T., Elo, M., Kiviranta, I., Dobai, J., Modis, L., Helminen, H.J., Arokoski, J.P., 1997. Specimen preparation and quantification of collagen birefringence in unstained sections of articular cartilage using image analysis and polarizing light microscopy. Histochem. J. 29 (4), 317-327.

Labus, K.M., Han, S.K., Hsieh, A.H., Puttlitz, C.M., 2014. A computational model to describe the regional interlamellar shear of the annulus fibrosus. J. Biomech. Eng 136 (5), 051009.

Marchand, F., Ahmed, A.M., 1990. Investigation of the laminate structure of lumbar disc anulus fibrosus. Spine 15 (5), 402-410.

Michalek, A.J., Buckley, M.R., Bonassar, L.J., Cohen, I., Iatridis, J.C., 2009. Measurement of local strains in intervertebral disc anulus fibrosus tissue under dynamic shear: contributions of matrix fiber orientation and elastin content. J. Biomech. 42 (14), 2279-2285.

Nachemson, A., 1960. Lumbar intradiscal pressure. Experimental studies on postmortem material. Acta Orthop. Scand. Suppl. 43, 1-104.

Natali, A., Meroi, E., 1990. Nonlinear analysis of intervertebral disk under dynamic load. J. Biomech. Eng. 112 (3), 358-363.

Nerurkar, N.L., Mauck, R.L., Elliott, D.M., 2011. Modeling interlamellar interactions in angle-ply biologic laminates for annulus fibrosus tissue engineering. Biomech. Model. Mechanobiol. 10 (6), 973-984.

Pezowicz, C.A., Robertson, P.A., Broom, N.D., 2006. The structural basis of interlamellar cohesion in the intervertebral disc wall. J. Anat. 208 (3), 317-330.

Preibisch, S., Saalfeld, S., Tomancak, P., 2009. Globally optimal stitching of tiled 3D microscopic image acquisitions. Bioinformatics 25 (11), 1463-1465.

Schollum, M.L., Robertson, P.A., Broom, N.D., 2009. A microstructural investigation of intervertebral disc lamellar connectivity: detailed analysis of the translamellar bridges. J. Anat. 214 (6), 805-816.

Showalter, B.L., Beckstein, J.C., Martin, J.T., Beattie, E.E., Espinoza Orias, A.A., Schaer T.P., Vresilovic, E.J., Elliott, D.M., 2012. Comparison of animal discs used in disc research to human lumbar disc: torsion mechanics and collagen content. Spine 37 (15), E900-E907.

Smith, L.J., Byers, S., Costi, J.J., Fazzalari, N.L., 2008. Elastic fibers enhance the mechanical integrity of the human lumbar anulus fibrosus in the radial direction. Ann. Biomed. Eng 36 (2), 214-223.

Ueno, K., Liu, Y.K., 1987. A three-dimensional nonlinear finite element model of lumbar intervertebral joint in torsion. J. Biomech. Eng. 109 (3), 200-209.

Urban, J.P., Roberts, S., 2003. Degeneration of the intervertebral disc. Arthritis Res. Ther 5 (3), 120-130.

Yu, J., Fairbank, J.C., Roberts, S., Urban, J.P., 2005. The elastic fiber network of the anulus fibrosus of the normal and scoliotic human intervertebral disc. Spine 30 (16), 1815-1820.

Yu, J., Tirlapur, U., Fairbank, J., Handford, P., Roberts, S., Winlove, C.P., Cui, Z., Urban, J., 2007. Microfibrils, elastin fibres and collagen fibres in the human intervertebral disc and bovine tail disc. J. Anat 210 (4), 460-471. 